\title{
Los caracteres adquiridos de Lukács. El problema de la inasunción biológica en las Humanidades, IV
}

\author{
Atafrascas Orbinio de la Estigia*
}

May 5, 2020

\begin{abstract}
I try to show in this brief note a simple case of the use of science with ideological, political or sectarian interests by those who, having prejudices, say they love and know it. That case, is the case of Lukács in his work on the criticism of irrationalism that emerges in the 18th and 19th century, in which he shows a deep scientific ignorance as well as a persistent and unpleasant defense of the ideology that he professes. If, someone, while affirming that science is valid knowledge using it in his speech, and his doctrine, advocates its use or considers science itself, burning the ship of demarcation, if, he maintains the recognized scientific methodology apart from his dogmas and arguments for being contrary to his creed and simultaneously ignoramus et ignorabimus the essential of contemporary scientific knowledge, then, the result of his convictions and litanies, with the emphasis or insistence that others allow him, contributes absolutely nothing to the science and confuses the clueless.
\end{abstract}

\section{Resumen}

Intento mostrar en esta breve nota un simple caso de la utilización de la ciencia con intereses ideológicos, partidistas o sectarios por aquellos que teniendo prejuicios dicen quererla y conocerla. Ese caso, es el caso de Lukács en su trabajo sobre la crítica al irracionalismo que emerge en los siglos XVIII y XIX, en el que muestra una profunda ignorancia científica al tiempo que una defensa pertinaz y desagradable de la ideología que profesa. Si, alguien, mientras afirma que la ciencia es un conocimiento válido utilizándola en su discurso, y su doctrina aboga por su uso o a ésta misma la considera ciencia quemando la nave de la demarcación, si mantiene la metodología científica al uso apartada de sus predios por ser contraria a su credo y simultáneamente ignoramus et ignorabimus lo esencial del conocimiento científico coetáneo, entonces, el resultado de sus convicciones y de sus letanías, con el énfasis o la insistencia que los demás le permitan, no aporta absolutamente nada a la ciencia y confunde al despistado.

\section{Introducción}

En las sociedades actuales complejas y densas el único pierde su propiedad, pierde el hecho de ser un individuo y disponer de ciertos grados de libertad

*Facultad de Filosofía A Fraja, Camiño Fondo, 7. Email: ng110777@gmail.com 
(Cf. Stirner, 2014 33). En un momento como el actual, donde la población mundial ronda lo 7.8 millardos y el alcance de los estados llega hasta los asuntos personales más nimios alcanzando el temible 'social caging 1

Las ideologías que necesitaban eliminar al diferente, al disidente, al incrédulo, al infiel, en fin, al otro, para mantenerse cohesionadas y llevar a cabo sus diseños delirantes, han obtenido su objetivo. Habiéndolo pretendido durante siglos pero por los medios equivocados, ahora se han topado con una solución inopinada que viene de la mano del número y de las vísceras, del deme y de la emoción. Del estado como estrategia, como condensación dinámica de las fuerzas políticas, como relación social, como el instrumento legitimador de la violencia (Véase, por ejemplo, VV. AA., 2009 [36]). Es este estado quien toma las decisiones por todos como si supiera lo que nos conviene a cada uno. Todo el planeta queda bajo el yugo de una idea que se extiende y se copia, un mundo feliz. Así, con cualquier excusa nos encierran y nos señalan que nada es de nuestra propiedad, que nada nos pertenece, ni siquiera nuestra volición. Nosotros comenzamos a ser el 'Nosotros' (Zamyatin, 1987 [38]). Nuestro pensamiento y nuestras vidas son suyas. Todo es un espectáculo, un realismo lukacsiano en el que no podemos participar. Da pánico escucharlos y a las mientes nos viene un, creemos, imposible, 'Nineteen Eighty-Four'. Nos encierran en nuestras, sus, casas y acabaremos dándoles las gracias. La famosa alienación es ahora tan mayúscula que ninguna artística semántica lukacsiana podría minorizarla ¿Qué está sucediendo realmente? Una parte es simplificable con pérdida de generalidad: La población mundial es tan ingente y sus movimientos suficientemente impredecibles, la tecnología tan eficiente para obtener información de cada individuo, y estos tan fácilmente manipulables a cambio del consabido bienestar del incapaz, que quienes ostentan el poder pueden legislar o cambiar las reglas como se les antojen. El poder del gobernante es el poder coercitivo, el uso de la fuerza bajo el paraguas de una consigna, la ilusoria legitimidad democrática. Y tú puedes hacer un diagnóstico pero no puedes curarte; puedes soñar, 'because reason must prevail' (Zamyatin, 1987: 322 38]), pero no tienes fuerza. Unos necios con su estulto paternalismo cretino obligan al individuo y doblegan su voluntad con caramelos emocionales. Las cosas no tienen remedio pero seguimos dispuestos a cambiarlas con buenas palabras. Otro idealismo.

Un pensador que pudo 'oler' esta evolución del estado benefactor al asfixiante, desde las primeras ideas marxistas del siglo XIX, fue Lukács. Nació en una familia judía y rica. Según sus palabras, familia sin interés en la religión pero sí con algún interés en el estatus económico e incluso aristocrático. Como muchacho era explícitamente contrario a las convenciones sociales y crítico con la disciplina materna y con la regla paterna de que el éxito es el criterio que de-

\footnotetext{
${ }^{1}$ Un vistazo y reflexión alrededor suele ser suficiente, pero si no, también puede leerse: 'the omnipotence of a nation-state in modernity is derived from its military might, economic control of material resources and ideological legitimacy. However, most of all its institutional supremacy is rooted in its territorialized organizational potency'; y la forma política dominante se convierte en el poder del estado, 'The ascendancy of the political arises from the state's monopolistic, centralized and institutionalized control over a particular territory. The steady rise of this administrative power of state is linked to the historical process of what Mann (1986: 112-114) calls social caging, whereby rulers have gradually imposed restrictions on individual freedoms in exchange for economic resources and political and military protection, in this way simultaneously generating mechanisms of social stratification and triggering the long term process of institutional and administrative centralization' (Malešević, 2009: 281 y ss. 22]). Claro como el agua.
} 
fine el haber actuado correctamente. Lector voraz desde los nueve años, la importancia de la literatura y la filosofía ${ }^{2}$ fue decisiva para un muchacho sin amigos en absoluto. Un tiempo después, el elitista, el 'Comrade Lukacs', era un 'people's commissar' de cuyas actividades como tal, él mismo dijo, 'there is little that can be said about that. At the time we all felt a bitter hatred for capitalism and all its forms. We wanted to destroy it at all costs and as quickly as possible. This unquestionably had an influence on the cultural policy of the party. Experiments were made which were fundamentally correct, but naive in their execution' (Lukács, 1983: 60 [20]). Y aun menos mal. Ahora, 'With the collapse of communism and the disintegration of Marxism in Western intellectual circles', parece hora de recuperar a Lukács para el pensamiento radical occidental, desde una perspectiva nueva, menos dogmática, menos de 'perro muerto', más filosófica y transgresora con la ideología (VV. AA., 2011 [37]). Pero para un lector desconfiado, no parece que haya mucho que salvar de su originalidad tan anclada en la importancia del partido. Si leemos que para Lukács, 'Technical, scientific and social development involves the deterioration of values, increased exploitation and inhumanity. Increasing rationality on the level of partial subsystems brings along the irrationality of the system as a whole. The development of capitalism projects the end of capitalism: the progress that creates the existential foundation of bourgeoisie leads to its fall as a class' (Kelemen, 2014: 74 [14]), entonces, me pregunto, ¿qué podemos salvar realmente? Está tan preocupado de mantener a flote su doctrina, tan neurótico con su defensa, tan cabizbajo al manejar la razón con un grado superlativo de dependencia, con la heteronomía que se le critica al contrario, que no estoy seguro de que podamos salvar algunos enseres por aquí o por alla

\section{Metodología}

Como es habitual he utilizado determinada bibliografía a mi alcance sin preocuparme de la exhaustividad, dado que Lukács es un autor al que se han dedicado centenares de artículos y un buen elenco de obras, tanto laudatorias, como críticas. En lo que sigue trataré de exponer:

(i) Un párrafo de Lukács. He elegido uno de su famoso libro 'Die Zerstörung der Vernunft' de 1954, traducido al español como 'El asalto a la razón', no la destrucción de la razón.

(ii) Causas que están en la raíz de sus expresiones y consecuencias que se derivan del párrafo lukacsiano.

(iii) Dos palabras sobre la ideología perniciosa para la ciencia por la que Lukács aboga continuamente.

(iv) Conclusiones sucintas.

(v) Agradecimientos.

\footnotetext{
${ }^{2}$ Para una biografía más académica véase Sziklai, (1993: 1-8 34]); también es muy útil, aunque de hace años, Mészáros, (1972: 153-200 24]).

${ }^{3}$ 'Not even a Marxism debased to an official creed will say much else, as witness the late Lukács' (Adorno, 2004: 376 [1]).
} 


\section{$3 \quad$ El hecho}

Lukács, escribe en 'El asalto a la razón':

'También en esto se revela claramente, el carácter fundamental del irracionalismo moderno. Al fracaso del tratamiento metafísico-mecánico de la dialéctica de la realidad, causa de la crisis general de las ciencias naturales en el período imperialista, no opone Bergson el conocimiento del movimiento dialéctico real y de sus leyes, pues esto sólo puede hacerlo el materialismo dialéctico. Bergson combate lo que hay de mecánico y de muerto en las doctrinas evolucionistas del tipo Spencer, pero a la par con ello niega, en biología, la herencia de los caracteres adquiridos. Por tanto, toma posiciones contra la teoría real de la evolución precisamente en el problema en que se planteaba la necesidad y la posibilidad de desarrollar dialécticamente las doctrinas de Darwin (como más tarde habrían de hacerlo Mitchurin y Lysenko, a base del materialismo dialéctico). Con lo cual su filosofía viene a entroncarse, ante todo, con aquel movimiento internacional encaminado a la destrucción de la objetividad de las ciencias naturales iniciado por Mach y Avenarius y que, en el período imperialista, encontró también representantes muy señalados en Francia; bastará con citar los nombres de Poincaré y Duhem.'

(Lukács, [1959] 1968: 22 [17])

Puede compararse la versión inglesa en (Lukács, 1981: 26 [18])

\subsection{Causas y Consecuencias}

En este fragmento, en el prefacio de su libro, en buena medida una declaración de intenciones resumida, Lukács menciona varias ideas que critica, y que es usual criticar en la ideología que él manifiesta, e incluso diríamos que hace bien en criticarlas pero, en realidad, son confusiones o ignorancia de nuestro pensador ¿Cómo no estar de acuerdo con él en su lucha contra el irracionalismo? Aunque en no todos los ámbitos, en ciencia el irracionalismo no puede formar parte de los contenidos, de los resultados; otra cosa es que determinado grado de irracionalismo haya sido útil heurísticamente en la investigación científica, que sirva o haya servido de camino, pero no de objetivo. El objetivo de la ciencia no es el irracionalismo. Y si no es su meta, tampoco su contenido, por extraño que pueda resultarnos a un ser vivo como nosotros. Se dirá, pero el libro de Lukács trata el irracionalismo desde Schelling y Hegel hasta Nietzsche y Heidegger. En efecto, y hay pasajes razonables. Sin embargo, la necesidad propagandística de adscribir su ideología a la ciencia, de hacerla pasar por ciencia, lo incita a comentar teorías que no entiende y a hacer afirmaciones que no pasan desapercibidas. Su libro no es una novela, ni siquiera una novela que analizar con su teoría sobre la novela. Es un libro de ensayo, de filosofía, que pretende ir más allá de los límites estrechos que le marca la razón. Buscando la destrucción de la de otros con la suya, que peca de lo mismo.

No voy a discutir aquí sobre cuestiones relativas a las interpretaciones correctas de conceptos marxistas, como cuando, 'In Lukács's hands alienation became a structural feature of the capitalist system of production and especially of social and political reproduction, rather than a superstructural reflection of the economic infrastructure, as in the conventional Marxist theory of ideology' 
(Aronowitz, 2015: 60 2]). Este tipo de discusiones para clarificar quién dijo qué en términos filosóficos, no son de mi interés en esta breve nota.

Lukács no tiene una definición de irracionalidad o racionalidad breve y general (Kelemen, 2014: 73 [14), entonces contra quién van sus críticas? ¿Simplemente se trata de desprestigiar a autores que supusieron, décadas después de su acmé, un epítome para las tendencias políticas que dieron lugar al nazismo y al fascismo? ¿A relacionar estos movimientos con los problemas sociales o con los cambios históricos? ¿Es que acaso no era consciente de que la irracionalidad que denosta conduce a la misma crítica de su crítica?

Vale. Pero, si podemos aceptar con cautela la actitud agonal contra el irracionalismo, no aceptamos el denominado por él, su opuesto, su racionalidad dialéctica ¿Qué es eso? ¿Qué es eso de una 'systematic Marxist dialectical Ontology'?; por este camino acabaremos leyendo la Lógica de Hegel para encontrar en ella soluciones a nuestros problemas prácticos. Lo que él denomina 'tratamiento metafísico-mecánico de la dialéctica de la realidad', es un sinsentido, un absurdo. No sabemos qué es la dialéctica de la realidad desde la ciencia; él tampoco. Y no sabemos cómo sería tratar algo metafísica-mecánicamente ¿Qué batiburrillo de tratamiento es éste? Si algo es metafísico, que va más allá de la física, que es trascendente o idealista en grado sumo, ¿cómo va unido a lo mecánico que por definición es indisoluble de lo material?

Según Lukács, Bergson combate lo que hay de mecánico y muerto en ciertas doctrinas evolucionistas pero con ello niega la herencia de los caracteres adquiridos, esto es, va contra la teoría real de la evolución '“Teoría real de la evolución'! Desde Marx, Lukács y similares se han visto compelidos a aceptar el darwinismo sobre la naturaleza pero a negar que las conclusiones del mismo afectasen al hombre directamente. Para éste tenemos al marxismo que debería desarrollar dialécticamente la teoría darwinista. 'Dialécticamente', no se nos olvide (Mészáros, 1972 24]). Obviamente, Bergson con su vitalismo y su idealista élan vital, no podía competir con los resultados darwinistas, y en cierto modo su irracionalismo e idealismo merecían la crítica lukacsiana. Pero no más.

Y a continuación, Lukács menciona el desarrollo dialéctico de las doctrinas de Darwin realizado por Mitchurin y Lysenko por medio, claro está, del materialismo dialéctico ¿Por qué utiliza a Mitchurin, si éste, aparte de ser un cultivador de nuevas variedades de frutales y flores, no publicó nada de interés y aún cuando lo hizo 'as has been repeatedly mentioned, Michurin rather poorly articulated his ideas' (Goncharov \& Savel'ev, 2016: 107 [10]). No hay ningún desarrollo del darwinismo efectuado con el materialismo dialéctico que aparezca en los libros de biología o genética usuales. Nada ¿Por qué? Porque la ideología marxista o comunista es insoluble en la ciencia, pues no es ciencia, sólo doctrina.

La ciencia que defiende Lukács es la ciencia de Terlestky contra la de Sájarov; Terlestky, el campeón de la pureza ideológica, un oculto de la KGB que niega la existencia de las fuerzas de inercia como fuerzas reales pues para él y su ideología son idealistas (Sájarov, 1990: 328 [32]). Es alucinante leer las memorias de Sájarov, donde relata a qué extremos de ignominia, maldad e ignorancia llegó la ciencia en Rusia hasta más allá de los años 60. Por eso, no me olvido de sus famosas y valientes palabras cuando en 1964, hirviéndole la sangre, y ante la elección de Nuzhdin 4 como miembro sénior de la Academia de Ciencias,

\footnotetext{
${ }^{4}$ Nuzhdin era uno de los colaboradores más cercanos de Lysenko y cómplice de la pseudociencia de éste y de la persecución de los genuinos científicos.
} 
tomó la palabra y dijo ante el estupor de muchos: '[Nuzhdin] Together with Academician Lysenko is responsible for the shameful backwardness of Soviet biology and of genetics in particular, for the dissemination of pseudoscientific views, for adventurism, for the degradation of learning, and for the defamation, firing, arrest, even death, of many genuine scientists' (Sájarov, 1990: 234 [32]). Esto no lo supo Sájarov de repente en 1964; desde 1920 era evidente que toda la biología soviética se había metido en un callejón ideológico basado en las memeces de los líderes mil veces repetidas o veneradas. Una de ellas se basaba en que el hombre nuevo se hace a sí mismo, es materia y como materia puede modelarse a voluntad. Si educamos a los niños con nuestra verdad conseguiremos esa sociedad comunista basada en la igualdad, sin propiedad privada, sin robos, sin delitos, sin sangre. Para eso el lamarckismo suena ideal, heredar un carácter que adquieres ontogenéticamente, esto es, en tu vida, en la escuela, de las palabras de los que saben. Da igual lo que diga la realidad, dan igual Darwin, Mendel y toda la escuela genética occidental con miles de artículos publicados revisados por pares. Cortemos las colas de los ratones. Sin atender a las pruebas, sin hacer ciencia, Lysenko desvariaba con sus experimentos y llevó a la agricultura soviética a la mayor y más profunda depresión y ulteriores hambrunas y a la biología a retroceder 200 años. Todo por una idea; una idea que nace en Marx y en sus discípulos. Darwin había descubierto la ley de la evolución en la naturaleza y Marx, decían, la ley de la evolución de las sociedades, del hombre. La incomprensión del mecanismo de la selección natural por estos pensadores les permitió seguir segregando al Homo sapiens del resto de la naturaleza. Como para los cristianos, el hombre es un caso aparte. Aquéllos que no sabían nada de biología, pontificaban con suficiencia sobre su objeto. Casi cien años después de la publicación del 'Origen' de Darwin, Lukács, el clérigo romántico, publica su asalto a la razón repitiendo las mismas tonterías de otrora. Las mismas. No hay excusa, lo que hubo es ideología nefanda, dogmatismo en los predios comunistas ¡Y lo hace Lukács en un trabajo que pretende atacar el irracionalismo que emerge de Hegel, Nietzsche, Heidegger,...!

Lukács se atreve a decir que los mitos y las mitificaciones ideológicas pretenden 'sugerir una concepción del mundo que corresponda a la de la filosofía del imperialismo allí donde la ciencia parece incapaz de ofrecer una visión de conjunto y reemplazar la perspectiva que ofrece la ciencia cada vez que ésta contradice la concepción propuesta por la filosofía paradójica del estadio del imperialismo'. Sustituyamos 'imperialismo' por lo que tenemos en mente. Disimulemos y volvamos 'oscuras las consecuencias sociales de las adquisiciones de la ciencia' (Lukács, 2000: 38 [21]). De eso se trata, de enfangarlo y confundirlo todo; no de ciencia, no de metodología científica, no de los contenidos de la ciencia ¿Cómo acaba su opúsculo sobre la filosofía burguesa? Con lo consabido, con lo esperable del dogma: 'Sólo el materialismo dialéctico es el que anima con vida real los problemas del nuevo mundo y los integra orgánicamente en su ideología' (Lukács, 2000: 55 [21]) Por eso, una vez que triunfa la revolución en Hungría y Lenin se regocija de cómo se llegó pacíficamente a ella, comienza el caos, la inflación, el terror y el resto de características habituales de la ideología. Sostenella y no enmendalla. Cuando esta revolución se establece, ¿a qué mente trastornada se le puede ocurrir establecer un 'National Council for the Products of the Mind'? A Lukács, un comisario político en cultura y educación; alguien que coloca 'flying book-stands' por todas partes donde se congregue gente para adoctrinarla, que fomenta un 'literary register' para encauzar la ambición de 
los jóvenes y no tanto, y, en una revista de la que es director, 'published their names, categorizing them along the lines of their political allegiance', con los comunistas a la cabeza. Y pagándoles por escribir lo que se esperaba de ellos. El jefe de Lukács en el comisariado, Kunfi, hubo de reconocer, 'the scientific, literary, and cultural life is unable to thrive without a certain atmosphere of freedom. During these ten weeks of the Proletarian dictatorship all intellectua1 and scientific life has noticeably vanished' ¿Acaso no se veía venir, Lukács? ¿Quién es realmente el idealista, quién el irracionalista, quién el inmoral? 'Kunfi complained of a lack of freedom under a dictatorship; but to make a dictatorship engender freedom is like trying "to forge an iron ring out of wood" (Zitta, 1964: 98-110 [39]). Estas lecturas resultan espeluznantes ¿Cómo es posible que una persona inteligente y culta pueda acabar haciendo las locuras que le conocemos? La respuesta en la biología.

Siempre encontraremos autores que pese a las evidencias insisten en defender lo indefendible, en arrastrar la ciencia por los silvales ideológicos, en tiznarlo todo de relativismo cambiando el significado de las palabras, 'La herencia de los caracteres adquiridos ('herencia con modificaciones' de Darwin) es el verdadero núcleo de la teoría de la evolución' (Olarieta, 2009: 11 [26]); si hay que defender a Lysenko, por motivos epistemológicos, léase políticos, porque es necesario cambiar el mundo, arrojar el capitalismo, el neoliberalismo o el imperialismo a las cárcavas de la historia, lo haremos sin ruborizarnos (por ejemplo, Cheroni, 2004 [4; Olarieta, 2009 [26]). No remaré en esas aguas oscuras. Terminaré con las críticas que Lukács hace a Mach, Avenarius, Poincaré o Duhem, por querer destruir la objetividad de las ciencias naturales ¿Cómo se puede ser tan sectario? ¿Es que acaso no conoce la obra de estos autores? Pueden ser atacados por múltiples motivos, a la escala del detalle, pero señalarlos como quienes pretenden eliminar la objetividad de la ciencia, es algo más que excesivo, fabuloso a secas. Tal vez Lukács vaya de eso en ocasiones, de fábulas.

\subsection{Dos palabras sobre la ideología lukacsiana}

La propaganda siempre ha sido un medio eficaz para manejar a los pueblos. La opinión política parece ser únicamente una cuestión de temperamento, como se deduce del contradictorio Manifiesto comunista y su 'revolutionary-utopian fantasies' (Conquest, 2000: 9, 34 [5]). La persecución del disidente, el control de la información que pueda llegar al ciudadano estableciendo que la correcta, que la verdadera es la oficial, la del gobierno, la del partido, la del tirano y denigrar el resto y en particular la simple crítica por ser falsa, interesadamente sesgada, burguesa, embustera que pretende con sus juicios y noticias derrocar o lastimar al régimer 5 ¿Cuándo ha cambiado esto? ¿Acaso hoy, que se escucha a los gobernantes decir que hay que restringir el derecho a la información privada, eliminar las mentiras por decreto? Acaso no se pueda ser más estúpido, pero sí se puede ser más malvado. Decir la verdad sin que la adulación la acreciente, u otro vano respeto la disminuya (Cervantes, 1936: 474 [3]).

Aunque a muchos les pareciera que 'the harshness of its methods seemed almost justified by the magnitude of its problems' (Schlesinger, 2019: cap. V, The Case of Russia [31]), cualquiera puede ver la ignominia de su sistema, un

${ }^{5}$ Naturalmente, hay muchas alternativas a mi descripción, en las que no me detengo. 
'régimen carcelario, ruinoso y genocida' (Jiménez, 2019: 35 13]), enraizado, como otros, en el animismo 'hors de la connaissance objective, hors de la vérité, étrangers et en définitive hostiles à la science, qu'ils veulent utiliser, mais non respecter et servir 6 (Monod, 1970:186-187 [25]), haciendo 'cosas extremadamente malvadas en el nombre del marxismo dogmático y doctrinario' (Dawkins, 2006: 278 6]). Si la cesta está al principio llena de buenas intenciones humanísticas, si de lo que se trataba era de 'humanizar al hombre', de barnizar a los más duros con el idealismo utilizado ad misericordiam, al poco, todo lo que quedó en la cesta fueron una hoz y un martillo como símbolos de los argumentos entonces usados ad baculum. De lo que se trataba era de 'acceder al cuarto trasero de la conciencia buenista' (Jiménez, 2019: 35 [13]).

Uno de los problemas que atraviesa toda la filosofía política es que incluso pensadores reflexivos y analíticos, como Bertrand Russell, terminan decantándose en cierto momento por alguna afirmación estulta abiológica como la que hizo refiriéndose a que la muerte de Lenin 'makes the world poorer by the loss of one of the really great men', al considerarlo el tipo de persona que, 'if the revolution succeeds, set to work to create a stable world in accordance with their conception' (Russell, 1970: 38 30]) ¡Con su concepción! Al contrario que Russell, espero que jamas ninguno de nosotros 'live to see his equal', fundamentalmente por el mesianismo o 'marxianismo' de un individuo que 'has as little love of liberty' (Russell, 1970: 42 [29]). Lo que leemos en el prefacio del libro de Russell, 'I cannot share the hopes of the Bolsheviks any more than those of the Egyptian anchorites; I regard both as tragic delusions, destined to bring upon the world centuries of darkness and futile violence', es lo correcto, lo que uno esperaría; y no por lo que vivimos hoy y lo que sabemos que sucedió. No dejan de resultar terroríficas la ingenuidad y candidez de Russell al escuchar las respuestas de un obcecado. Hay que contestar con fuerza ante la actitud pusilánime, ante la derrota anticipada. Rescato por ello mismo, y para hacer algo de justicia a Russell, lo que dice éste de Lenin en el prólogo, acaso habiendo meditado mejor las respuestas que recibió en su momento: 'He is a man who entertains a number of elaborate and dogmatic beliefs -such as philosophic materialism, for example- which may be true, but are not, to a scientific temper, capable of being known to be true with any certainty' (Russell, 1970: 8 [29]).

La revolución rusa no liberó a los trabajadores, no los hizo partícipes del colectivismo burocrático en que se había convertido el ideal comunista (Schlesinger, 2019: cap. V, The Case of Russia 31]). Otra vez, todo para el pueblo pero sin el pueblo. Acabaron siendo el tipo de mercancia que el marxismo condenaba en las sociedades capitalistas. La instrumentalización del individuo, del obrero, del proletariado fue rápidamente asumida y constante a lo largo del tiempo tras el éxito de la revolución de 1917. La persecución del disidente primero por agitprop, y luego por medio de rehenes, ostracismo, reclusión, cárceles o muerte, está documentada ampliamente. Las entrevistas y opiniones de varios ex-comunistas nos dejan un panorama de lo sucedido, una visión sobre la ingenuidad del hombre y sobre su maldad que nos vuelven absolutamente pesimistas (Véase, por ejemplo, VV. AA., 1963 [35]). En realidad, ya parece imposible la existencia

\footnotetext{
6 'fuera del conocimiento objetivo, fuera de la verdad, extraños y en definitiva hostiles a la ciencia, que quieren utilizar, mas no respetar y servir', pág. 161. Ediciones Orbis, traducción de Francisco Ferrer Lerín, revisada por el prof. Antonio Cortés Tejedor.
} 
de gobiernos limitados en cuanto estos se basan en constituciones democráticas. Probablemente no dejan de ser contradictorias, como había notado Gödel respecto de la americana, incluso afectadas de inconstitucionalidad por ello mismo (Hoppe, 2011: 267 y ss. [12]), como para que aquéllos estén en manos de líderes iluminados, mesías y profetas de nuevas viejas religiones.

Lenin utiliza un trozo de la carta de Lasalle a Marx (24/06/1852) como dedicatoria en su libro, 'Qué hacer': el partido se fortalece depurándose. Eso de depurarse o purgar siempre nos lleva a una enfermedad o presencia de toxinas o similares. Hay que curar. Sin embargo, fuera hace mucho frío o quien se mueve no sale en la foto y, aunque Lukács tenía cosas que decir tras la muerte de Lenin, en particular algunas buenas apostillas filosóficas a las tendencias burocráticas y a las intenciones más que obvias de actuación, sin embargo y quién sabe si por aquéllo mismo, inspirado en la revolución bolchevique, en el alcance internacional del movimiento y en su propia insistencia en la centralidad de la organización política, 'Lukács remained in the fold' (Aronowitz, 2015: 61 [2]). Quién lo hubiese dicho, se hizo estalinista al calor de la Realpolitik de éste, y, si el mundo capitalista era relativamente estable habría que reconsiderar y reformar las tesis de 'History and Class Consciousness' (Löwy, 1979: 193 y ss. [16]).

\subsection{Conclusiones}

Del maestro del marxismo europeo, Lukács, dicen 'under no conditions whatsoever will the ideologist renounce his ideology (Feuer, 2010: 105 [7]) ¿Qué pasaría si algún marxista ortodoxo tuviese delante la prueba empírica de que todas las afirmaciones hechas por Marx fuesen falsas? 'Even if this were to proved, every serious orthodox marxist would still be able to accept all of Marx's theses in toto without having to renounce his orthodoxy for a single moment' (Lukács, 1972: XXVI, 1 [19]). Asombrosa la fe del párroco. O como dice con la misma generalidad Sacristán, tomándonos el pelo, quizás haya 'proposiciones marxianas que se presten más o menos a la duda o al rechazo, ya sea por crítica de su consistencia interna, ya por insuficiente base empírica', ya por poco pertinentes, pero en todo caso deben tratarse como condicionales contrafácticos, esto es, no es necesario considerarlas falsas (Rendón, 1983: 10, entrevista a Sacristán [28]). Asombrosos estos diáconos irredentos. Estas tonterías hablan por sí solas. Como resumen del texto de nuestro autor, concluyo:

(i) La actitud de Lukács es anticientífica. No le interesa la ciencia nada más que para servirse de ella cuando es compatible con lo que previamente cavila o dogmáticamente asume. Actúa críticamente con los resultados científicos que comprometen sus afirmaciones, rechazándolos en contra de toda evidencia. Así, para nuestro caso y otros muchos, la necia percepción de que es posible diseñar un hombre sin conocerlo, un hombre nuevo, a la medida de nuestras concepciones y que idealmente cualquiera de esos diseños locos o sandios puede llevarse a la práctica mediante la educación o la coerción, conduce irremediablemente a Lamarck, el asidero de los torpes miopes que no terminan de comprender que el Homo sapiens es un ser vivo con una historia que importa, la filogenética. Ya se nos ha recordado más de una vez que comenzando por Marx $[7$ y luego sus epígonos, el interés por

\footnotetext{
${ }^{7} \mathrm{Al}$ decir de Sacristán, Marx conocía superficialmente la ciencia de su época salvo la que
} 
la verdad científica siempre ha quedado en segundo plano, supeditado a la ideología producto de alguna ebullición cerebral, al rezo de un catecismo simplista (Politzer, 1976 [27]).

(ii) ¿Qué concepto de hombre maneja Lukács? ¿A qué galimatías lingüístico debemos referirnos como a su Homo sapiens? Dar nuevos significados a una palabra no desvela automáticamente la verdad del referente 8 . En el confuso texto de Merleau-Ponty (1970: 160 [23]), refiriéndose a Lukács, se indica que para éste el concepto de hombre debe ser en sí mismo dialéctico. Y si por 'hombre queremos decir naturaleza o atributos positivos', entonces Lukács lo rechazaría. Claro, el hombre es algo más que la suma de sus partes, no es sólo biología, sino dialéctica. Pero decir esto, es no añadir nada relevante a lo que se pregunta. Para decirlo con Merleau-Ponty, 'si uno entra en el relativismo lo suficientemente profundo, alcanzamos dentro de él su propia trascendencia'. Interprétese a voluntad. Se entiende en parte esta precaución de cocinar a su gusto algunos de sus propios términos; en caso contrario estaríamos hablando de biología, fisiología, ecología o disciplinas afines de las que lo ignoran todo.

(iii) Mientras que el científico está preparado para considerar que el holismo es inviable, que es una metodología errónea para conseguir teorías falsables de la realidad, el marxismo, 'the ideologist, as a myth-maker', estima que la primacia de los motivos económicos en la explicación histórica no son lo que 'constituye la diferencia decisiva entre el marxismo y la ciencia burguesa, sino el punto de vista de la totalidad' (Feuer, 2010: 130-131 7]). Es decir, el hombre debe ser comprendido en su totalidad. Esto es, debemos aceptar una reificación de las cualidades y habilidades humanas en tanto que, según Lukács, no forman parte del todo orgánico que somos (Lukács, 1972: 100 [19) $)^{9}$ Esto ya viene de una desfachatez, de una inversión de los valores científicos, de llamar ciencia a lo que no lo es; ahí tenemos a Lenin quejándose por los ataques al socialismo y preguntándose si '¿se han levantado voces en contra de la ley constitucional que garantiza

estaba relacionada con la agricultura. Había sido un gran lector de Liebig y de las investigaciones de éste sobre el ciclo de los nutrientes en el suelo, pero, siempre es posible afirmar que 'the wider social implications were most penetratingly examined by Karl Marx' (Foster \& Bellamy, 1998: 43 8). Las conclusiones de estos autores ignorando la población del planeta en 1998 y dando pábulo a aforismos triviales sobre la tierra, como, 'sustaining the earth, as Marx wrote, "as the inalienable condition for the existence and reproduction of the chain of human generations"', (Foster \& Bellamy, 1998: 57 [8]) merecería un comentario más extenso.

${ }^{8}$ 'The dichotomy of subject and object is not to be voided by a reduction to the human person, not even to the absolutely isolated person. The question of man, a question whose present popularity extends all the way to Marxism of the Lukács persuasion, is ideological because its pure form dictates the invariant of the possible answer, even if that invariant is historicity itself' (Adorno, 2004: 51 [1]). Tampoco Adorno nos explica nada con eso de que la cuestión del hombre es ideológica.)

${ }^{9}$ Para una versión moderna y abstrusa del aspecto político del concepto de reificación de Lukács, obsérvese: 'One politically crucial aspect of the theory of reification is its critical analysis of depoliticization implicit to the specific ontology of the commodity' (Hartle, 2017: 21 [1]; También, Gandesha \& Hartle, 2017 [9]. O bien este otro: 'There is a good deal of irony in the fact that the brutal and primitive functionaries who more than forty years back damned Lukács as a heretic, because of the reification chapter in his important History and Class Consciousness, did sense the idealistic nature of his conception' (Adorno, 2004: 190 [1]). Adorno siempre consideró que Lukács había confundido reiteradamente reificación con objetivación. 
la libertad de ciencia y de investigación científica en la mayoría de los países europeos' (Lenin, 2010: 17 [15]). Hay que ignorar qué es la ciencia, definirla a tu complacencia y paladar o tener caradura para asimilar una determinada ideología a la ciencia.

(iv) Aunque no dejo de aceptar de Lukács la 'representative monumentality of his undertaking', (Mészáros, 1972: 95 [24]), al leerlo uno tiene la sensación de derrota previa y de anacronismo, de que sus textos terminan de aclararnos la dificultad mayúscula de volver a reunir y mantenerlas reunidas, quién lo diría, en la misma amalgama mena y ganga, no necesariamente respectiva, filosofía política y praxis política. Otra vez lo de Platón. Basta de pensar y actuemos, pero actuemos según pensamos. A base de aforismos hueros memorizables no se solucionan los problemas que el entorno plantea. Y la ciencia en sus contenidos no es buena amiga del especulador. Como era esperable, Lukács o los adláteres marxistas no consiguen que la ciencia sea el nexo de unión entre su pensamiento y la realidad.

\subsection{Agradecimientos}

Son varios compañeros, amigos y algo más que amigos que a lo largo del tiempo me ayudaron con sus juicios, comentarios y críticas de esta historiografía a no sincopar mis lecturas para una apreciación y perspectiva óptimas, según ellos. Escuché, pero por displicencia severa no hice mucho caso. Sin embargo, sí a un niño que en una encrucijada me indicó, hace muchos años, el sendero que llevaba a la Bahía.

\section{Bibliografía}

[1] Adorno, Theodor W. Negative Dialectics. Taylor \& Francis e-Library, 416 pp., [1973] 2004.

[2] Aronowitz, Stanley. Against Orthodoxy: Social Theory and Its Discontents. Palgrave Macmillan, New York, 189 pp., 2015.

[3] Cervantes Saavedra, Miguel. El ingenioso hidalgo Don Quijote de la Mancha. Ramón Sopena editor, Barcelona, 892 pp., 1936.

[4] Cheroni, Alción. El caso Lisenko: una relectura. Llull, vol. 27, 609-629 pp., 2004.

[5] Conquest, Robert. Reflections on a Ravaged Century. W.W. Norton \& Company, New York, 319 pp., 2000.

[6] Dawkins, Richard. The God delusion. Hougton Mifflin Company, Boston, New York, 406 pp., 2006.

[7] Feuer, Lewis S. Ideology and the ideologists. Transaction Publishers, New Brunswick, 219 pp., [1975] 2010.

[8] Foster, John Bellamy \& Magdoff, Fred. Liebig, Marx and the Depletion of Soil Fertility: Relevance for Today's Agriculture. Monthly Review, 50(32): 43-60 pp., 1998. 
[9] Gandesha, Samir \& Hartle, Johan F. The Spell of Capital: Reification and Spectacle. Amsterdam University Press, 224 pp., 2017.

[10] Goncharov, N.P. \& Savel'ev, N.I. Ivan V. Michurin: On the 160th Anniversary of the Birth of the Russian Burbank. Russian Journal of Genetics: Applied Research, Vol. 6(1): 105-127 pp., 2016.

[11] Hartle, Johan F. Reification as Structural Depoliticization: The Political Ontology of Lukács and Debord. In Gandesha, Samir \& Hartle, Johan F. The Spell of Capital: Reification and Spectacle. Amsterdam University Press, 2136 pp., 2017.

[12] Hoppe, Hans-Hermann. Democracy. The God That Failed. The Economics and Politics of Monarchy, Democracy, and Natural Order. Transaction Publishers, New Brunswick, London, 304 pp., 2011.

[13] Jiménez Losantos, Federico. Memoria del comunismo. De Lenin a Podemos. La Esfera de los Libros, S.L., Madrid, versión electrónica, 753 pp., 2018.

[14] Kelemen, János. The Rationalism of Georg Lukács. Political Philosophy and Public Purpose. Series Editor: Michael Thompson. Palgrave Macmillan, New York, 144 pp., 2014.

[15] Lenin, Vladimir Ilich. ¿Qué hacer? Problemas candentes de nuestro movimiento. Ministerio del Poder Popular para la Comunicación y la Información, Caracas, 351 pp., 2010.

[16] Löwy, Michael. Georg Lukács. From Romanticism to Bolchevism. NLB, London, 219 pp., 1979.

[17] Lukács, Georg. El asalto a la razón. La trayectoria del irracionalismo desde Schelling hasta Hitler. Ediciones Grijalbo S.A., Barcelona, México, 707 pp., [1954] 1968.

[18] Lukács, Georg. The destruction of Reason. Humaninties Press Inc., Atlantic Highlands N.J., 865 pp., 1981.

[19] Lukács, Georg. History and Class Consciousness: Studies in Marxist Dialectics. The MIT Press, Cambridge, 356 pp., [1968] 1972.

[20] Lukács, Georg. Record of a Life. An Autobiographical Sketch. Edited by Istvan Eörsi. Translated by Rodney Livingstone. Verso, 204 pp., [1971] 1983.

[21] Lukács, Georg. La crisis de la filosofía burguesa. Ediciones Elaleph.com, 55 pp., 2000.

[22] Malešević, Siniša. Collective Violence and Power. In The SAGE Handbook of Power. Edited by Stewart R. Clegg \& Mark Haugaard. SAGE, London, Los Angeles, 274-291 pp., 2009.

[23] Merleau-Ponty, M. Western Marxism. Telos, 6: 140-161, 1970.

[24] Mészáros, István. Lukács' Concept of Dialectic. The Merlin Press, London, 211 pp., 1972. 
[25] Monod, Jacques. Le hasard et la nécessité. Essai sur la philosophie naturelle de la biologie moderne. Editions du Seuil, Paris, 219 pp., 1970.

[26] Olarieta Alberdi, Juan Manuel. El linchamiento de Lysenko. Nómadas. Revista Crítica de Ciencias Sociales y Jurídicas. Universidad Complutense, Madrid, última edición: 19 de diciembre de 2008, 96 pp., 2009.

[27] Politzer, Georges. Elementary principles of philosophy. International Publishers, New York, 171 pp., [1975] 1976.

[28] Rendón Alarcón, Jorge. Manuel Sacristán habla con dialéctica. Entrevista con Manuel Sacristán Luzón. Dialéctica N¹3: 97-119, 1983. Disponible online. Acceso, 27/04/2020: aquí.

[29] Russell, Bertrand. The Practice and Theory of Bolshevism. George Allen \& Unwin Ltd., London, 188 pp., [1920] 1921.

[30] Russell, Bertrand. Lenin: An Impression. Australian left Review, AprilMay, 38-39 pp., [1924] 1970.

[31] Schlesinger, Arthur Meier. The Vital Center. The Politics of Freedom. Routledge, Oxon, New York, versión epub [300pp.], [1949] 2017.

[32] Andrei Sakharov. Memoirs. Translated from Russian by Richard Lourie. Alfred A. Knopf, New York, 776 pp., 1990.

[33] Stirner, Max. El Único y su propiedad. Editorial Sexto Piso, Madrid, 452 pp., 2014.

[34] Sziklai, Lászlo. György Lukács: A Biography. In Hungarian Studies on György Lukács. Vol. I. Edited by Lázslo Illés, Farkas József, Miklós Szabolcsi, István Szerdahelyi. Akadémiai Kiadó és Nyomda Vállalat, Budapest, 1-8 pp., 1993.

[35] VV. AA. The God that Failed (Arthur Koestler, Ignazio Silone, André Gide, Richard Wright, Louis Fischer, Stephen Spender). Richard Crossman, Editor. Harper Colophon Books, Harper \& Row, Publishers, New York, 273 pp., [1949] 1963.

[36] VV. AA. The SAGE Handbook of Power. Edited by Stewart R. Clegg \& Mark Haugaard. SAGE, Los Angeles, 484 pp., 2009.

[37] VV. AA. Georg Lukács Reconsidered. Critical Essays in Politics, Philosophy and Aesthetics. Edited by Michael J. Thompson. Continuum International Publishing Group, London, 253 pp., 2011.

[38] Zamyatin, Yevgeny. We. Avon Books, New York, 232 pp., [1920] 1987.

[39] Zitta, Victor. Georg Lukacs'Marxism, Alienation, Dialectics, Revolution. A study in Utopia and Ideology. Introduction by Harold D. Lasswell. Springer Science+Business Media B.V., The Hague, 305 pp., 1964. 